\title{
Suicide attempt among adolescent in Tamil Nadu: A case-control study
}

\author{
Vinoth Krishna Dass ${ }^{1}$, Arun Prasanna Chandrasekaran ${ }^{2}$, Vinayagamoorthy Venugopal ${ }^{3 *}$, Asokkumar Muthu ${ }^{4}$, \\ Gandhibabu Ramanathan 5
}

\begin{abstract}
${ }^{\mathbf{1}}$ Associate Professor, ${ }^{\mathbf{2 , 3}}$ Assistant Professor, ${ }^{4}$ Professor and Head, ${ }^{\mathbf{5}}$ Professor, ${ }^{\mathbf{1 , 2 , 4 , 5}}$ Dept. of Psychiatry, ${ }^{3}$ Dept. of Community Medicine, ${ }^{1,3}$ Sri Manakula Vinayagar Medical College \& Hospital, Puducherry, ${ }^{2}$ Government Theni Medical College, Theni, Tamil Nadu, ${ }^{4,5}$ Rajah Muthiah Medical College and Hospital, Annamalai University, Tamil Nadu, India
\end{abstract}

*Corresponding Author: Vinayagamoorthy Venugopal

Email: drvinayagamoorthy@gmail.com

\begin{abstract}
Introduction: Suicide is a complex social issue and a serious global health problem. Its incidence among adolescents is increasing. Understanding of risk factors helps early detection and prompt treatment of high-risk youngsters.

Materials and Methods: It was a hospital-based case-control study carried out among 60 adolescent suicide attempters and 60 of their normal counterparts for the period of one year. Pretested structured questionnaire was used to collect information. Ethical principles were followed throughout the study. SPSS version 24 was used to do the analysis. Odd's ratio (OR) and its $95 \%$ confidence interval (CI) were calculated as a measure of the strength of association between risk factors and suicide attempts.

Results: Among the study participants, $70 \%$ were in the age group of $16-18$ years and $45 \%$ were males. The identified risk factors for suicide attempt were, presence of psychiatric illness (OR-7.8; 95\% CI: 3.3-19), substance abuse among family members (OR-4.3; 95\% CI: 2-9.3), family history of attempted suicide (OR-7.2; 95\% CI: 1.5-3.3), family history of completed suicide (OR-3.6; 95\% CI: 1.2-1.1) and residence at rural areas (OR-2.8; 95\% CI: $1.2-6.3)$. Suicide intent score was significantly correlated with various standard scoring tools for stress and depression among cases.

Conclusion: The established risk factors should be considered in creating an effective intervention program and strategies with intersectoral collaboration and coordination to prevent suicide among high-risk adolescents.
\end{abstract}

Keywords: Adolescent, Attempted suicide, Case-control study, Risk factors.

\section{Introduction}

Suicide is a complex social issue and a serious public health problem. Suicidal behavior is a spectrum that ranges from suicidal ideation at one end to completed suicide at the other end. World Health Organization reports that every year nearly 800,000 deaths occur due to suicide worldwide. ${ }^{1}$ Suicide is the second leading cause of death among youngsters (15 to 29 years old) globally and its incidence rate is increasing. ${ }^{2}$ More than $78 \%$ of global suicides occur in low and middle-income countries. ${ }^{3}$ In India, over one lakh lives are lost every year due to suicide and in the last three decades, the suicide rate was found to be increased by $43 \% .^{4}$ The National Crime Record Bureau, report reveals that South Indian states has the highest suicide rate among India and Tamil Nadu stands in third position with 18.6/100,000 population. $^{5}$

As there is no effective protocol to predict suicide among adolescents in routine clinical practice, improving the recognition and understanding of clinical, psychological, sociological, and biological factors could help in the detection of high-risk youngsters so as to assist in treatment options. Therefore the present study was designed with the objectives to assess the association of childhood adversity, demographic factors and psychiatric co-morbidities with adolescent suicide attempt. We also tried to identify the correlation between suicide intent score with stressful life events, hopelessness and depressive scores among suicide attempters.

\section{Materials and Methods \\ Study setting and design}

Our study was an age and gender-matched case-control study that was carried out in a tertiary care teaching hospital situated in Cuddalore district of Tamil Nadu. It is a 1000 bedded teaching hospital. The average outpatient and inpatient load of Psychiatry department per day is 80 and 20 respectively. The department admits and provides care to nearly 150 attempted suicide patients every year.

\section{Study population}

Cases were adolescents belonged to both genders and in the age group of 13 to 18 years, admitted with the history of attempted suicide in the emergency department and were referred to the Department of Psychiatry for providing care and support during the year 2012. Subjects with the stable physical condition who could undergo detailed assessment were included. Those who were in the state of disorientation and confusion which interfered with the administration of rating scale to them and those without a reliable informant were excluded. Controls were the adolescents who accompanied those patients admitted to any other departments of the hospital with complaints other than the psychiatric disorder. They were selected in such a way their age and gender were matched with the selected cases.

\section{Sampling and sample size}

Sample size was calculated to be 60 cases and 60 controls using OpenEpi software version 3.0, taking the exposure of cases to worrying issues in family as $65 \%$ and exposure of controls to same risk factor as $35.3 \%$ and odds ratio of 3.4 , 
based on previous study ${ }^{6}$ with $95 \%$ confidence interval and $90 \%$ power. Expected number of cases in a year based on previous records is around 150 in numbers. Cases fulfilling the eligibility criteria were consecutively selected into the study till the calculated sample size was reached during the data collection period of one year. Once a case was selected control was selected in the ratio of $1: 1$ as per the eligibility criteria.

\section{Study tool and study variables}

Pretested structured proforma was developed for collecting information on socio-demographic details like age, gender, education, occupation, socio-economic status and marital status. Socio-economic status was decided based on modified Kuppuswamy's scale for urban residence and BG Prasad's scale for the rural setting, for the year 2012. ${ }^{7}$ Information on any past history of physical illness and the previous attempt at suicide among participants was collected. History of selected childhood adversities like substance abuse among family members attempted and completed suicide in family members was recorded. Cases and controls were clinically examined for any psychiatric morbidity and diagnosis were made according to ICD-10. The degree of suicidal intent of cases was measured with the help of Suicide Intent Scale (SIS) developed by Beck (1979). ${ }^{8}$ For both cases and controls, Presumptive Stressful Life Events Scale (PSLES), ${ }^{9}$ Hopelessness Scale (HS) by Beck (1974) ${ }^{10}$ and Hamilton Depression Rating Scale $(\mathrm{HAM}-\mathrm{D})^{11}$ were administered. PSLES is used to capture the stressful life events in the past one year. It is simple to use, can be administered to both, literate and illiterates, and is designed for use in Indian population. HS by Beck (1974) is used to assess the spectrum of negative attitudes. HAM-D (1960) is used to assess the most important symptoms of depressive disorders. The severity of their depression is rated by probing mood, feelings of guilt, suicide ideation, insomnia, agitation or retardation, anxiety, weight loss, and somatic symptoms.

\section{Study procedure and data collection}

Cases and controls were selected as per the selection criteria mentioned above. Then interview was conducted with the study subject to obtain information on risk factors using predesigned pilot-tested questionnaire. Their family caregivers were also interviewed as and when required. It was carried out in two to three sessions, each consisting of forty-five minutes to one hour. Age and gender-matched controls were recruited from medical and surgical wards as and when a case was registered to the study. They were also interviewed using the similar proforma adopting same methods and following ethical principles similar to that of cases.

\section{Ethical consideration}

Institute Human Ethics Committee clearance was obtained before initiating the study procedure. As participants were adolescent assent were obtained from them and the parental consent were also obtained from their parents. All ethical principles have adhered throughout the study.

\section{Data entry and statistical analysis}

Data were entered into EpiInfo software version 7.2.2.6 and analyzed using SPSS software version 24. Description of categorical variables was done in frequency and proportion. Chi-square test was used to find out the association between various risk factors and suicide attempt. The Pearson correlation coefficient was used to correlate suicidal indent score with other scores. Binary logistic regression was carried out with those risk factors that were significantly associated with suicide attempt in bivariate analysis to find out the adjusted Odds ratio (OR), a measure of the strength of association and presented with its $95 \%$ confidence interval (CI). All tests were two-tailed and p-value $<0.05$ was considered statistically significant.

\section{Results}

Thirty percent of both cases and controls were in the age group of $13-15$ years and remaining were in the age group of $16-18$ years. Male constituted $45 \%$ of the participants in both case and control group and the rest were females. Among cases $13(21 \%)$ were illiterate and only 4 (6.7\%) had studied above high school level of education. Among controls $4(6.7 \%)$ of them were illiterate and $10(16.7 \%)$ had studied above high school level of education. Majority of the participants were the student by occupation in both groups, $32(53.3 \%)$ in cases and $30(50 \%)$ in control group. $15 \%$ of the cases and $8.3 \%$ of the controls were unemployed. As per their residence status, $81.7 \%$ of the cases and $61.7 \%$ of the controls were from the rural setting. According to modified socio-economic scale for the year $2012,81.7 \%$ of the cases and $83.3 \%$ of the controls were from lower (Class-V) category. Majority of them were unmarried in both groups. Among the cases, 35 (58.4\%) had some psychiatric illness, whereas among controls only nine $(15 \%)$ had psychiatric morbidity. The details of which were presented in the Table 1.

The association of various clinic-social factors with the attempt to commit suicide was shown in Table-2. Among those who stayed in the rural area, 49 (57\%) had attempted suicide whereas of those who stayed in urban areas, 11 $(32.4 \%)$ had attempted suicide. The odds of staying in the rural area were 2.8 times statistically more among those who attempted suicide compared to those who had not attempted it. Subjects belonged to higher SES had got 1.1 times higher risk of attempting suicide than those from lower SES but this was not statistically significant $(\mathrm{p}=0.81)$. Similarly unmarried adolescent had 1.8 times higher risk of attempting suicide than their married counterparts but this association was again not statistically significant. No one from case group had any current medical illness because of which odds ratio could not be computed. Subjects with any psychiatric illness had 7.2 times significantly higher chance to attempt suicide than those without any psychiatric morbidity. Among the participants who gave the positive history of substance abuse in the family, 42 (66.7\%) attempted suicide and $21(33.3 \%)$ did not. Those who gave the positive family history of addictions were 4.3 times at higher risk of attempting suicide compared to those who 
Table-1: Socio-demographic details and psychiatric morbidity of study participants

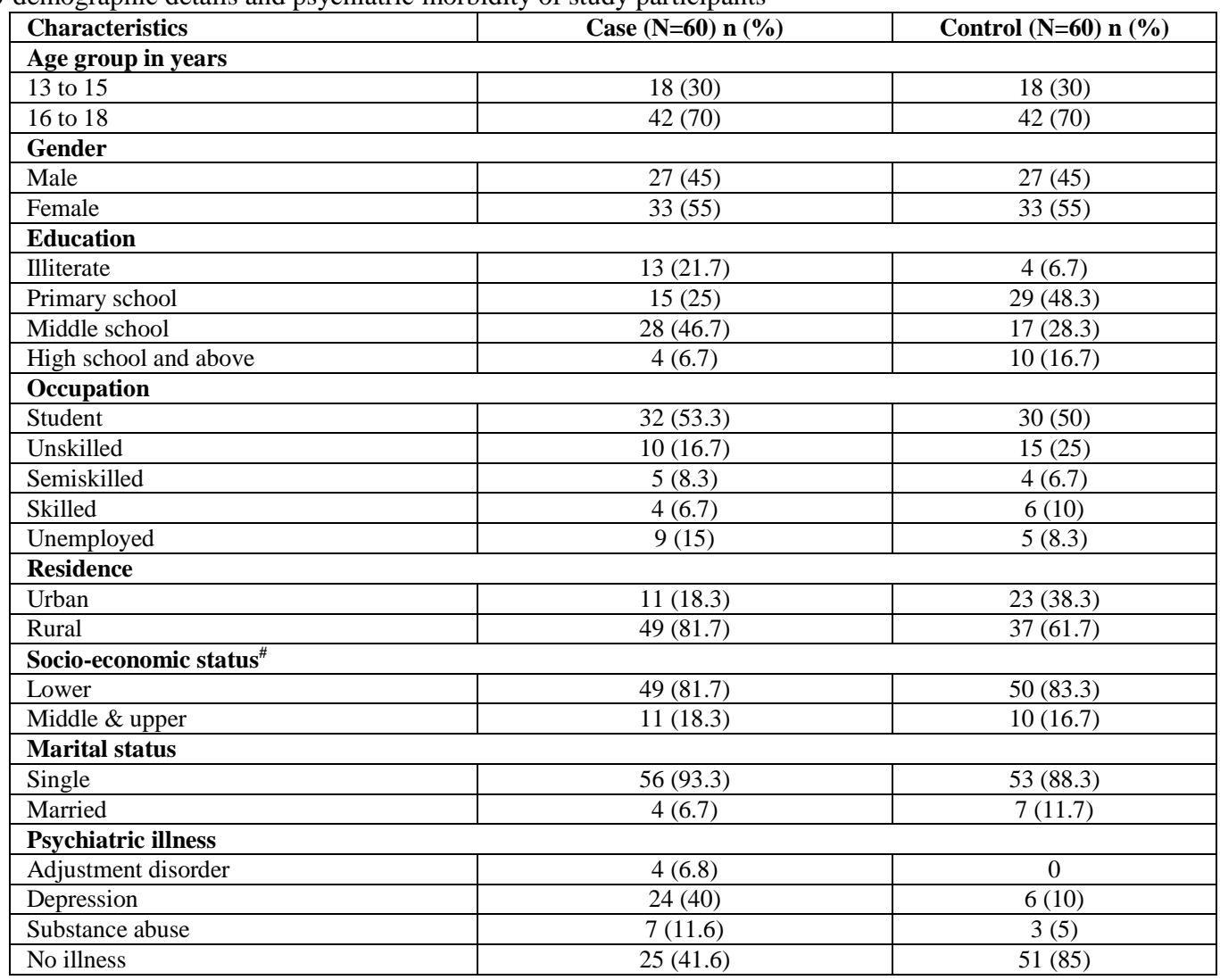

Note: \# Based on Kuppuswamy's classification for urban and BG Prasad scale for rural residence modified for the year 2011.

Table-2: Association of childhood adversities and various clinco-social risk factors with attempt of suicide

\begin{tabular}{|c|c|c|c|c|c|}
\hline Features & $\begin{array}{c}\text { Cases } N=60 \\
n(\%)\end{array}$ & $\begin{array}{c}\text { Control } \mathbf{N}=\mathbf{6 0} \\
n(\%)\end{array}$ & $\mathrm{X}^{2}$ value, $\mathrm{df}$ & p value ${ }^{\#}$ & OR $(95 \% \mathrm{CI})^{\$}$ \\
\hline \multicolumn{6}{|l|}{ Residence } \\
\hline Urban & $11(32.4)$ & $23(67.6)$ & \multirow[t]{2}{*}{$5.91,1$} & \multirow[t]{2}{*}{$0.01 *$} & 1 (Reference) \\
\hline Rural & $49(57)$ & $37(43)$ & & & $2.8(1.2-6.3)$ \\
\hline \multicolumn{6}{|l|}{ Socio-economic status } \\
\hline Low & $49(49.5)$ & $50(50.5)$ & \multirow[t]{2}{*}{$0.05,1$} & \multirow[t]{2}{*}{0.81} & 1 (Reference) \\
\hline Middle \& High & $11(52.4)$ & $10(47.6)$ & & & $1.1(0.4-2.8)$ \\
\hline \multicolumn{6}{|l|}{ Marital status } \\
\hline Single & $56(51.4)$ & $53(48.6)$ & \multirow[t]{2}{*}{$0.90,1$} & \multirow[t]{2}{*}{0.34} & $1.8(0.51-6.6)$ \\
\hline Married & $4(36.4)$ & $7(63.6)$ & & & 1 (Reference) \\
\hline \multicolumn{6}{|l|}{ Physical illness } \\
\hline Yes & $3(42.8)$ & $4(57.2)$ & \multirow[t]{2}{*}{$0.15,1$} & \multirow[t]{2}{*}{0.69} & $0.74(0.15-3.4)$ \\
\hline No & $57(50.4)$ & $56(49.6)$ & & & 1 (Reference) \\
\hline \multicolumn{6}{|l|}{ Psychiatric illness } \\
\hline Yes & $35(79.5)$ & $9(20.5)$ & \multirow[t]{2}{*}{$24.2,1$} & \multirow[t]{2}{*}{$0.001^{*}$} & $7.8(3.3-19)$ \\
\hline No & $25(32.9)$ & $51(67.1)$ & & & 1 (Reference) \\
\hline \multicolumn{6}{|c|}{ Family history of substance abuse } \\
\hline Yes & $42(66.7)$ & $21(33.3)$ & \multirow[t]{2}{*}{$14.7,1$} & \multirow[t]{2}{*}{$0.001^{*}$} & $4.3(2-9.3)$ \\
\hline No & $18(31.6)$ & $39(68.4)$ & & & 1(Reference) \\
\hline \multicolumn{6}{|c|}{ Family history of attempted suicide } \\
\hline Yes & $12(85.7)$ & $2(14.3)$ & \multirow[t]{2}{*}{$8.1,1$} & \multirow{2}{*}{$0.004^{*}$} & $7.2(1.5-33)$ \\
\hline No & $48(45.3)$ & $58(54.7)$ & & & 1 (Reference) \\
\hline \multicolumn{6}{|c|}{ Family history of completed suicide } \\
\hline Yes & $15(75)$ & $5(25)$ & \multirow[t]{2}{*}{$6.0,1$} & \multirow[t]{2}{*}{$0.01^{*}$} & $3.6(1.2-10)$ \\
\hline No & $45(45)$ & $55(55)$ & & & 1 (Reference) \\
\hline \multicolumn{6}{|l|}{ Previous suicide } \\
\hline Yes & $4(100)$ & 0 & \multirow[t]{2}{*}{$4.1,1$} & \multirow[t]{2}{*}{$0.04 *$} & \multirow[t]{2}{*}{ Not applicable } \\
\hline No & $56(48.3)$ & $60(51.7)$ & & & \\
\hline
\end{tabular}

Note: $X^{2}$-Chi square value, , \# $p$ value based on Chi square test, ${ }^{*}$ statistically significant ( $p$ value <0.05), \$OR-Odds Ratio, CI-Confidence interval. 
Table-3: Comparison of scores of mental status among study groups using various standard tool

\begin{tabular}{|l|c|c|c|c|}
\hline Tool used & $\begin{array}{c}\text { Cases } \\
\text { N=60 } \\
\text { Median (IQR) }\end{array}$ & $\begin{array}{c}\text { Control } \\
\text { N=60 } \\
\text { Median (IQR) }\end{array}$ & $\begin{array}{c}\text { Mann-Whitney } \\
\text { test statistics }\end{array}$ & $\begin{array}{c}\text { p value } \\
\#\end{array}$ \\
\hline Presumptive Stressful Life Events Scale & $79.4(58.3)$ & $17(26)$ & 585 & $<0.001^{*}$ \\
\hline Hopelessness scale & $4.8(4.7)$ & $0.48(1.5)$ & 513 & $<0.001^{*}$ \\
\hline Hamilton Depression Rating Scale & $9.1(8.2)$ & $1.2(3.8)$ & 569 & $<0.001^{*}$ \\
\hline
\end{tabular}

Note: \# Based on Mann-Whitney test statistics, IQR-Inter quartile range, *statistically significant.

Table 4: Correlation between suicide intent score with various standard scoring tools among cases

\begin{tabular}{|l|c|c|}
\hline Tool used & Pearson's correlation co-efficient & p value\# \\
\hline Presumptive Stressful Life Events Scale & 0.74 & $<0.001^{*}$ \\
\hline Hopelessness scale & 0.76 & $<0.001^{*}$ \\
\hline Hamilton Depression Rating Scale & 0.79 & $<0.001^{*}$ \\
\hline
\end{tabular}

Note: \# Based on Pearson's correlation statistics, *Statistically significant.

Table 5: Adjusted analysis showing association between various risk factors and adolescent suicide attempt

\begin{tabular}{|l|c|c|}
\hline Risk factors & $\begin{array}{c}\text { Adjusted OR } \\
\text { (95\% CI) }\end{array}$ & p value \\
\hline Family history of substance abuse & $3.8(1.2-11.8)$ & $0.02^{*}$ \\
\hline Presumptive Stressful Life Events Scale score & $1.03(1.01-1.05)$ & $0.004^{*}$ \\
\hline Hopelessness scale score & $1.39(1.01-1.96)$ & $0.05^{*}$ \\
\hline
\end{tabular}

Note: OR-Odd's Ratio, CI- Confidence Interval, * Statistically significant, Nagelkerke's $R$ square value was 0.68 for the adjusted logistic regression model.

have no such history in the family (p-value 0.001 ). The odds of attempting suicide was 7.2 and 3.6 times higher among those who had the positive history of attempted suicide and positive history of completed suicide in their family respectively, compared to those who had negative histories. These strengths of associations were statistically significant too. Among those who attempted suicide four of them had the positive history of previous suicide attempts and among controls, none had such history. The median score of cases that explains their mental status in terms of depression, hopeless attitude, and stressful life event using standard tools were significantly higher than the controls (Table 3 ). Suicidal indent score was strongly correlated with stressful life events, hopelessness and depressive scores with the correlation coefficient of $0.74,0.76$ and 0.79 respectively among cases. These correlations were positive and also statistically significant (Table 4).

Binary logistic regression analysis carried out with those risk factors found statistically associated and correlated with adolescent suicide attempt showed that only three factors were independently associated with suicide attempt. They were the positive family history of substance abuse, increasing PSLES score and HS score. These findings were statistically significant (Table 5).

\section{Discussion}

The risk factors identified in the current study for attempting suicide among adolescents were the history of substance abuse, attempted suicide and completed suicide in the family. Other significant risk factors associated with the risk of suicide attempt were the rural residence and psychiatric illness among subjects. Stressful life events, hopelessness and depressive scores of cases were significantly higher than controls. Stress, hopelessness, and depression score were strongly and significantly correlated with suicide intent score among cases.

In the present study adolescents residing in the rural area were found at more risk for the suicide attempt than the urban residents (OR: 2.8; 95\% CI: $1.2-6.3$ ). The study done in the United States also conveyed the similar result. ${ }^{12}$ The possible explanation could be less availability and accessibility of health services in rural areas especially mental health services, low mental health literacy, low family income, less recreational facilities, lesser penetration of modern technologies and the difference in the method of suicide in terms of easy availability and accessibility of various methods. Cultural factors also play a significant role in the observed rural-urban disparity in terms of the helpseeking behavior, and the stigma associated with mental illness.

The childhood related risk factors identified from the previous studies for suicide attempts were the history of past suicide attempt, substance abuse, psychiatric disorder, serious physical illness, history of suicides in family or friends, and parents not living together. ${ }^{13-15}$ All these studies were cross-sectional in nature. Though they established the association between those risk factors and suicide attempt, they could not estimate the strength of association between those risk factors and suicide attempts. The present study also identified similar risk factors but our study was casecontrol in nature that usually generates higher evidence than cross-sectional study.

A study done in Shimla found adolescents with worrying issues in the family had adjusted OR of 2.5 to attempt suicide. ${ }^{6}$ A study done in Delhi among adolescents reported adverse family events like fight among parents (OR-1.6; 95\% CI: 0.9-2.8), physical abuse by parents (OR1.5 ; $95 \%$ CI: $1.1-2.1$ ), neglected by parents (OR-1.8; $95 \%$ CI: 1.1-3.2), not enjoying time spent with parents (OR-0.9; 95\% CI: $0.5-1.4$ ), severe financial problems in family (OR- 
1.1; 95\% CI: $0.7-1.6)$ as risk factors. ${ }^{16}$ The current study also captured that adverse family events in the form of substance abuse (adjusted OR-3.8; 95\% CI: 1.2 - 11.8), attempted suicide (OR-7.2; 95\% CI: 1.5-33), completed suicide (OR-3.6; 95\% CI: 1.2-10) among family members were associated with risk of suicide attempt among adolescent.

A cohort study done in Sweden showed childhood adversities like death in family (OR-1.9; 95\% CI: 1.3-2.8), suicide in family (OR-2.9; 95\% CI: 1.4-5.9), parental substance abuse (OR-1.9; 95\% CI: 1.4-2.4), parental psychiatric disorder (OR-2.0; 95\% CI: 1.5-2.8), substantial parental criminality (OR-2.3; 95\% CI: 1.7-3.0) and parental separation or single parent (OR-1.4, 95\% CI: 1.2-1.7) were associated with suicide attempts among young adults. ${ }^{17} \mathrm{~A}$ case-control study in Australia also confirmed that family history of substance abuse had an OR of 3.21 with $95 \%$ CI of 1.51-6.82 for attempting suicide among adolescent. ${ }^{18}$ Studies done in France and Iran also revealed that adverse family events had the higher risk for suicide attempt. ${ }^{19,20}$ These findings are again in alignment with the current study findings.

The meaning of suicidal thoughts and adolescence normative attitudes towards suicide varies across diverse cultural, religious and economic settings. ${ }^{21}$ Hence the family environment where the adolescent is exposed to various socio-cultural factors plays a vital role in creating and shaping the thoughts of suicide. This could be the reason for higher suicide attempt among adolescent exposed to the adverse family situation. It is consistently evident that genetic factors play an important role in the predisposition to suicide and suicidal behaviors. ${ }^{22}$ It is possible that part of the predisposition to suicide may be transmitted via the presence of impulsive and impulsive-aggressive behaviors from parents to children. Some molecular genetic studies support the role of some genes that code for components of the serotonergic pathway in the etiology of suicidal behavior. ${ }^{23}$ This could be a possible reason for the observed higher risk for suicide attempt among adolescent who had the positive family history of completed and attempted suicide in the present study.

In the current study subjects with psychiatric illness has 7.8 times higher risk of attempting suicide (95\% CI: 3.3-19). The similar finding was recorded in a case-control study in Australia (OR: 3.1 to 18.7$).{ }^{18}$ Cross-sectional studies from Atlantic region of the United States (OR-1.7; 95\% CI: 1.3, $2.1),{ }^{24}$ and New Zealand ${ }^{25}$ also identified psychiatric illness as the risk factor for suicide attempt. Previous studies showed lower SES was associated with suicide attempt but in the present study middle and the high-income group had a higher chance (OR-1.1; 95\% CI: $0.4-2.8$ ) to attempt suicide. But the finding was not statistically significant. Stressful life events, hopelessness, and depression were more among those who attempted suicide in the present suicide and this finding was consistent with previous studies. ${ }^{4,26,27}$ The transition from childhood to adolescence, which in itself is accompanied by physical and psychological challenges such as depression, anxiety, and loneliness. During this transition period if other risk factors especially family psychopathologies occur concurrently then it could lead to suicidal behaviors among them.

\section{Strengths and Limitations}

In the current study apart from identifying the association between risk factors and suicide attempt, we estimated the strength of association as well. The interview to identify the risk factors happened over a period of two days and this reduced the chance of information bias by the patients. Some information on risk factors was cross-checked with the parents and this reduced the reporting bias. Hospitalbased studies usually suffer generalization of findings as the participants are not real representatives. In the current hospital-based study, those cases of attempted suicide who had developed serious complications were excluded hence the selected cases may not be the real representative of all attempted suicide adolescents admitted in the hospital.

\section{Conclusion and Recommendations}

In conclusion, this study identified children from rural areas, exposed to childhood adversities, suffering from mental illness were associated with an increased risk for suicide in adolescent period. Hence it is important to understand the social mechanisms of mental health morbidity and suicide in order to develop effective interventions, aiming to alleviate the risk of suicide in children. Those identified risk factors need to be the specific target for prevention of national policies and strategic plans related to suicide and mental health. Professionals like general practitioners, pediatricians, school teachers, school counselors, social workers and social psychologists need to be trained in identifying depression and suicidal behaviors in adolescents. If required they can refer them to a mental health professional or mental health service for thorough assessment and effective management. Suicide is preventable; hence a social and public health approach created by the integration of stakeholders across multiple levels within society including the individual, the family, the community, and the health care system is to be developed.

\section{Source of Funding}

None.

\section{Conflict of Interest}

None.

\section{References}

1. World Health Organisation: Suicide [Internet]. World Health Organ. [cited 2018 Mar 9];Available from:

http://www.who.int/mediacentre/factsheets/fs398/en/

2. Cash SJ, Bridge JA. Epidemiology of Youth Suicide and Suicidal Behavior. Curr Opin Pediatr. 2009;21(5):613-9.

3. Turecki G, Brent DA. Suicide and suicidal behaviour. Lancet Lond Engl. 2016;387(10024):1227-39.

4. Vijayakumar L. Indian research on suicide. Indian J Psychiatry. 2010;52(Suppl1):S291-6. 
5. National Crime Records Bureau (NCRB). Accidental deaths and Suicide [Internet]. 2010 [cited 2020 Feb 20]; Available from: www.ncrb.nic.in

6. Thakur D, Gupta A, Thakur A, Mazta S, Sharma D. Prevalence and predictors of suicidal ideations among school going adolescents in a hilly state of India. Ind Psychiatry $J$. 2015;24(2):140.

7. Singh T, Sharma S, Nagesh S. Socio-economic status scales updated for 2017. Int J Res Med Sci. 2017;5(7):3264

8. Beck AT, Kovacs M, Weissman A. Assessment of suicidal intention: the Scale for Suicide Ideation. J Consult Clin Psychol. 1979;47(2):343-52.

9. Singh G, Kaur D, Kaur H. Presumptive stressful life events scale (psles) - a new stressful life events scale for use in India. Indian J Psychiatry. 1984;26(2):107-14.

10. Beck AT, Weissman A, Lester D, Trexler L. The measurement of pessimism: the hopelessness scale. J Consult Clin Psychol. 1974;42(6):861-5.

11. Hamilton M. A Rating Scale for Depression. J Neurol Neurosurg Psychiatry. 1960;23(1):56-62.

12. Fontanella CA, Hiance-Steelesmith DL, Phillips GS, Bridge JA, Lester N, Sweeney HA, et al. Widening rural-urban disparities in youth suicides, United States, 1996-2010. JAMA Pediatr. 2015;169(5):466-73.

13. Kumar CS, Chandrasekaran R. A study of psychosocial and clinical factors associated with adolescent suicide attempts. Indian J Psychiatry. 2000;42(3):237.

14. Dutta S, Gupta SD. Demographic factors associated with suicidal ideation among college students in Kolkata, India. Indian J Health Wellbeing. 2014;5(7):46.

15. Kar N. Profile of risk factors associated with suicide attempts: A study from Orissa, India. Indian J Psychiatry. 2010;52(1):48.

16. Sidhartha T, Jena S. Suicidal behaviors in adolescents. Indian $J$ Pediatr. 2006;73(9):783-8.

17. Björkenstam C, Kosidou K, Björkenstam E. Childhood adversity and risk of suicide: cohort study of 548721 adolescents and young adults in Sweden. BMJ. 2017;j1334.

18. Page A, Morrell S, Hobbs C, Carter G, Dudley M, Duflou J, et al. Suicide in young adults: psychiatric and socio-economic factors from a case-control study. BMC Psychiatry. 2014; 14(1):68.
19. Larosa E, Consoli SM, Hubert-Vadenay T, Leclesiau H. Factors associated with suicidal risk among consulting young people in a preventive health center. L'Encephale. 2005;31(3):289-99.

20. Golshiri P, Akbari M, Zarei A. Case-control study of risk factors for suicide attempts in Isfahan, Iran. Int $J$ Soc Psychiatry. 2017;63(2):109-14.

21. Oppong Asante K, Kugbey N, Osafo J, Quarshie EN-B, Sarfo JO. The prevalence and correlates of suicidal behaviours (ideation, plan and attempt) among adolescents in senior high schools in Ghana. SSM - Popul Health. 2017;3:427-34.

22. Zai CC, de Luca V, Strauss J, Tong RP, Sakinofsky I, Kennedy JL. Genetic Factors and Suicidal Behavior [Internet]. In: Dwivedi Y, editor. The Neurobiological Basis of Suicide. Boca Raton (FL): CRC Press/Taylor \& Francis; 2012. Available from: http://www.ncbi.nlm.nih.gov/books/NBK107191/

23. Turecki G. Suicidal behavior: is there a genetic predisposition? Bipolar Disord. 2001;3(6):335-49.

24. Arria A, O'Grady K, Caldeira K, Vincent K, Wilcox H, Wish E. Suicide ideation among college students: A multivariate analysis. Arch Suicide Res. 2009;13(3):230-46.

25. Fleming TM, Merry SN, Robinson EM, Denny SJ, Watson PD. Self-reported suicide attempts and associated risk and protective factors among secondary school students in New Zealand. Aust N Z J Psychiatry. 2007;41(3):213-21.

26. Morano CD, Cisler RA, Lemerond J. Risk factors for adolescent suicidal behavior: Loss, insufficient familial support, and hopelessness. Adolescence 1993;28(112):851.

27. Shain BN. The Committee on Adolescence. Suicide and suicide attempts in adolescents. Pediatr. 2007;120(3):669-76.

How to cite this article: Dass VK, Chandrasekaran AP, Venugopal V, Muthu A, Ramanathan G. Suicide attempt among adolescent in Tamil Nadu: A case-control study. Telangana J Psychiatry. 2020;6(1):58-63. 